\title{
OPTIMAL TREATMENT STRATEGIES FOR MUSCULOSKELETAL DISEASES
}

\author{
KJETIL K. HAUGEN
}

\begin{abstract}
This paper presents a model which aims to find an optimal treatment time for patients with musculoskeletal diseases. The stochastic dynamic programming model presented is based on minimizing expected costs given a stochastic processes for cure with and without treatment as well as costs for treatment and reduced quality of life as a consequence of the disease.

The main results derived in the paper include explicit conditions for an interior optimal solution and a simple and intuitive approximate solution.

Even though the presented model is specifically aimed at musculoskeletal diseases, the model's generality should make it applicable for a wide range of disease treatment strategies.
\end{abstract}

\section{INTRODUCTION}

\subsection{Health care expenditures}

The recent years have shown an explosive rise in expenditures on health care. During the period from 1965 to 1986, the overall U.S. expenditures on health care rose from $5.9 \%$ to $10.8 \%$ of GNP [9]. The reasons for such a development are not very hard to understand. Today, expensive and advanced operations such as bypass operations and heart transplants are performed routinely - unthinkable only a few years ago.

In other western economies such as the Norwegian, where health care is largely viewed as a public good, free of charge for the public, one would expect even more dramatic cost changes. This as a result of both the recent improvements in medical technology but also as a result of another important effect - the general demographic trend of ageing population.

Surely, as Figure 1 indicates, The ministry of Health and Social Affairs (SHD) shows a substantial position in Norwegian economy with it's share of expenditures of $34.4 \%^{1}$.

As a result of the development described above and a clear notion of a foreseeable future with growing problems of such a kind, SHD has initiated various

$M S C$ (2010): primary 90C39, 90C90.

Keywords: musculoskeletal disease, optimal treatment policy, interior optimum, bang-bang solutions, stochastic dynamic programming.

Financial support from the Norwegian Ministry of Health and Social Affairs is gratefully acknowledged.

${ }^{1}$ Updated numbers as of today (2013) indicates a continuing trend with similar expenses amounting to more than $50 \%$ of the share of total expenditures [10]. 


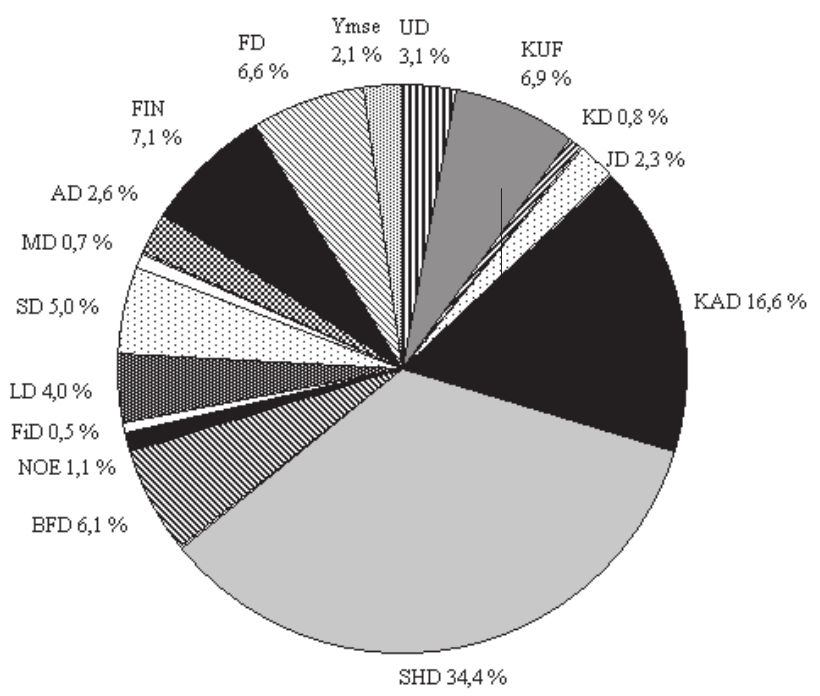

Figure 1. Expenditure share in 1996 for various Norwegian Ministries.

research projects with efficiency improvements in the health sector as the typical objective. This paper is written as a result of such a project and deals with a certain disease category - musculoskeletal diseases.

\subsection{Musculoskeletal diseases}

The diseases within the category musculoskeletal diseases are normally defined as all diseases and suffering within the human muscular and skeleton system. The typical diseases within this category are Repetitive Strain Injuries (RSI) and Rheumatic diseases.

In Norway, a large share of expenditures within the health care system may be addressed to this category. According to a white paper by the Norwegian Parliament [6], $46 \%$ of long time and $20 \%$ of short time sickness absence are caused by musculoskeletal diseases.

As sickness absence constitutes a large share of total $\operatorname{costs}^{2}$ within the sector, it seems sensible to attack this category in order to obtain cost reduction measures.

\subsection{Modelling aspects of musculoskeletal diseases}

Musculoskeletal diseases have some characteristics which make them special. Obviously, for almost all diseases the causality between treatment and disease progression may be uncertain. That is, it may be hard to predict if a treatment will cure the disease and if it does, when. However, musculoskeletal diseases seem from a medical point of view - to be harder both to diagnose correctly and cure than most other disease categories.

\footnotetext{
${ }^{2}$ According to [6] and other sources [3,8], a reasonable estimate on costs related to musculoskeletal diseases amounts to 20 bill. NOK or more than 3 bill. US\$.
} 
There may be distinct medical reasons for this, but other important explanations may exist. The medical fact that such diseases are hard to diagnose and cure may influence people to "choose" such diseases as substitutes for other problems. High age combined with today's not very "elderly-friendly" labour market and the possibility of obtaining reasonable pensions may lead certain people to choose a diagnosis instead of unemployment.

Some evidence supporting such a hypothesis exists. A public program referred to as "social insurance and rehabilitation program" [7] reports that in various projects, with the objective of bringing people back from social security and into the labour market, the clients did not take much interest in participation. They report that in certain projects, more than $60 \%$ of the clients refused to participate.

This evidence does of course not necessarily mean that a significant share of people diagnosed as patients with musculoskeletal diseases are trying to "cheat" the system. However, a hypothesis of the type described above is not weakened by such findings.

Anyway, the reason for bringing such socio-economic arguments into this study, is to justify some special model considerations used later on - namely the existence of a possibility of being cured from a musculoskeletal disease without treatment. Obviously, it is possible to become cured from almost any disease without treatment. Presumably, stories of miraculous treatment from much more serious diseases than those depicted here are known to the reader. Hence, it should not be hard to accept the existence of a probability of "self-treatment" for almost any disease. However, the reasoning above is introduced in order to argue for the fact that the probability of recovering from musculoskeletal diseases untreated, not only exists, but it may in some instances be of a magnitude not much smaller than the probability of recovering from the disease with treatment. Given the existence of people who have chosen the diagnose without being ill, the probability of recovering without treatment may even exceed the probability of recovering with treatment. It is plausible to imagine that a treatment program introduced to a person who actually knows that he does not have the disease (and wants to stay "ill") may prove to be more inefficient than leaving the person alone.

\section{THE MODEL}

Due to the large costs involved with treatment of patients with musculoskeletal diseases in Norway, and the above discussed hypothesis of possible "self-cure"; the following questions regarding today's treatment strategy were formulated:

- Does today's treatment strategy involve treatment of the correct patients?

- Given that a patient is to be treated, is the timing correct?

The first question implies that certain patients may as well recover from their "illness" without treatment. Obviously, a lack of treatment for such patients imply cost efficiency. The second question is essentially just a more detailed version of the first one; saying that some persons may benefit from being treated earlier than today's schedule.

To sum up: We may believe in the following trade-off situation. Introducing an earlier time of treatment may lead to reduced costs involved with an effective cure 
or rehabilitation. On the other hand, treating patients who get cured by themselves leads to inefficient use of treatment costs. The model formulated below balances such a trade-off.

\subsection{Basic assumptions}

The model described below may be summed up as follows: The model aims to find an optimal time for treatment of patients with a musculoskeletal disease. The optimal timing decision is based on a finite time horizon, a set of conditional probabilities for cure with or without treatment, a treatment cost structure and a disease cost structure. The model minimizes expected costs.

\subsection{Symbols}

The following symbols are used in the model:

$T:$ Time horizon

$t:$ Time period subscript, $t \in\{1,2 \ldots, T\}$

$p_{t}$ : Probability of being cured in period $t$ after treatment in period $t-1$

$q_{t}$ : Probability of being cured in period $t$ without treatment in period $t-1$

$c_{t}$ : Treatment cost in time period $t$

$k_{t}$ : The cost incurred during a time period with illness

As the symbols defined above show, the necessary data to the model consists of two stochastic processes (the vectors $p_{1}, \ldots, p_{T}$ and $q_{1}, \ldots, q_{T}$ ) and two cost vectors $c_{1}, \ldots, c_{T}$ and $k_{1}, \ldots, k_{T}$. Note that this definition implicitly introduces some significant assumptions - state independence for instance. However, a discussion on these topics will be skipped for now ${ }^{3}$.

The content of the two probability vectors should be fairly comprehensible, but the two cost vectors might require some more discussion. The treatment costs $\left(c_{1}, \ldots, c_{T}\right)$, are meant to reflect the actual costs of the treatment. In Norway, with a small private health-market, they may be hard to estimate, but they should be composed of the costs associated with usage of medical personnel and infrastructure involved in the treatment. The other cost component $\left(k_{1}, \ldots, k_{T}\right)$, may be harder to imagine. The basic point is the assumption of a cost associated with staying ill. This cost may be measured at an individual level including the mental and physical strain resulting from the disease and lost earnings as a result of absence on sick leave. In such a case, the model should be viewed as an individual decision model. Alternatively, one might try to measure this cost in a socio-economic frame. Then, components such as pension effects, effects on governmental budgets and so on should be added.

Surely, such a cost is hard to measure in any environment - socio-economic or decision theoretic.

\subsection{Decision tree}

The decision tree in Figure 2 shows the main elements of the decision model.

\footnotetext{
${ }^{3}$ Some further remarks on these assumptions will be given in Subsection 2.5.
} 


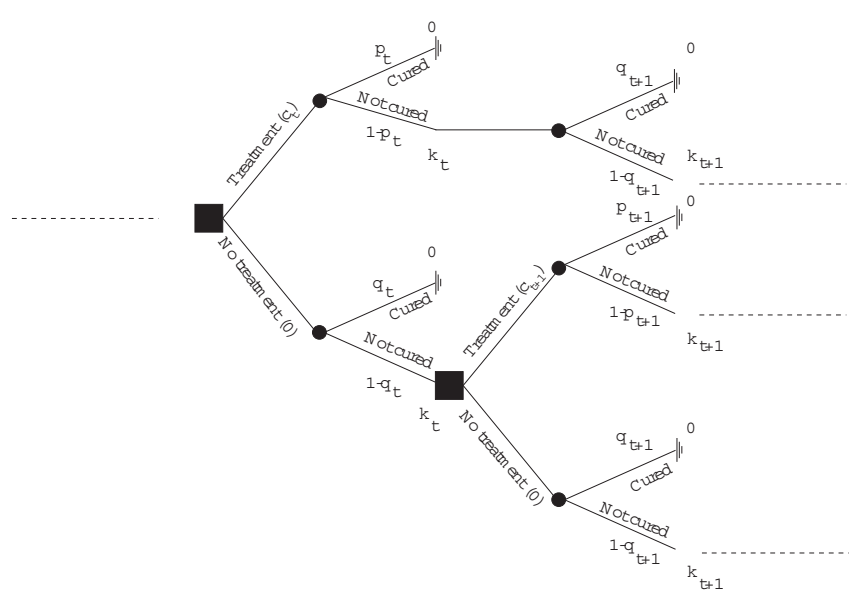

Figure 2. The decision problem depicted by a decision tree.

In a given time period $t$, a decision on whether to treat or not will have to be made. After this decision, "nature" decides the outcome for the patient - cured or not cured. Note that the patient, as discussed above, may get cured without treatment. Given treatment in period $t$, the probability of being cured is $p_{t}$, while the probability of being cured without treatment is $q_{t}$. The cost $c_{t}$ accrues in period $t$ if the patient is treated. No treatment implies no cost -0 in the decision tree. If the patient leaves period $t$ not cured, the cost $k_{t}$ accrues. Note that if a person has been treated, it is assumed that another treatment is illegal in this model. The reason for this assumption is discussed in Subsection 2.5. (This assumption is depicted with grounding symbols in the decision tree.) As no other costs accrues when a person is cured, it is unnecessary to expand the decision tree after this instance.)

\subsection{Similar modelling in the literature}

The basic source for similar modelling seems to be the two Israeli scientists Gafni and Mehrez $[2,4,5]$, who, in a series of articles in the mid eighties, treated the problem of finding the optimal timing of a surgery for a non-fatal disease.

Their work was inspired by micro-economic life-insurance modelling by Yaari [13] and Barro et al. [1] in the seventies. The analogy between optimal treatment timing and life insurance may need some more explanation. The basic problem for the individual involved with accepting or rejecting a life insurance policy may be that of timing. Should the policy be accepted today or should the acceptance be postponed. Surely, the event of death is not assumed influence able, but the consequence is. Hence, an individual faces the decision of rejecting the policy now, yielding one set of consequences if death occurs or accepting it (and the policy costs) but different (and better) consequences if death occurs.

Gafni and Mehrez are using a maximal expected utility framework in their models. They assume that an individual is facing the decision of undertaking surgery which may "cure" a disease (or not). They assume the existence of an underlying 
probability mechanism for the individuals probability density function of surviving $t$ more years without surgery and a corresponding density given surgery. (These two mechanisms correspond to $q_{t}$ and $p_{t}$ in 2.2.) The surgery is assumed costless, hence the basic trade off is that of choosing between continuing life with reduced life quality or gambling on a successful surgery.

One of the basic results of Gafni and Mehrez is the existence of a "bang-bang" solution under fairly general assumptions. The term "bang-bang" solution refers to a "now or never" optimal strategy. That is, an interior optimal solution does not exist and the surgery is either conducted immediately or never.

They also discuss which assumptions need to be altered in order to obtain an interior optimal solution, but they fail to give some direct mathematical results needed to find the solution. Section 3 will show similar characteristics in this model but also some stronger results on how to obtain interior optimal solutions.

\subsection{Some alternative modelling considerations}

As discussed in Subsection 2.2, the model briefly outlined by Figure 2 implicitly makes some important assumptions. This section will discuss these assumptions in a greater depth.

2.5.1. State dependency. According to Figure 2, the stochastic mechanisms and cost structures are assumed independent of earlier actions. That is, the probability of being cured without treatment does not depend on any earlier attempts to cure the disease. Surely, this may be questionable. Likewise, the cost associated with staying ill $\left(k_{t}\right)$ is assumed unaffected by a previous attempt to cure the disease. It seems obvious that these elements may be affected by unsuccessful treatments. Note, however, that the modelling structure does not block the possibility of building such mechanisms into the model. The results obtained in Section 3 may, however, be severely affected.

2.5.2. Time horizon - one treatment. Another important assumption is that of allowing only one attempt to treat the disease. This is obviously not even close to a practical situation ${ }^{4}$, but the main point in this model has been to look at a treatment timing problem. That is, it is assumed that the time horizon is sufficiently short to assume that it is impossible (or impractical) to impose several treatments. Again, the modelling frame (so far) does not really block this possibility, but again, the ease of obtaining solutions may be severely affected.

2.5.3. Number of possible decisions - outcomes. Another obvious assumption is that of the number of outcomes and treatment strategies. The decision variables are assumed to be binary. That is, a treatment is either initiated or not. It is surely possible to choose among a broader set of alternatives. Similarly, it is clear that the outcomes (CURED, NOT CURED) may be modelled in a more detailed way. These situations may be handled (with some added complexity) with the means of Section 3.

\footnotetext{
${ }^{4}$ It is not common try only one treatment on a disease if the initial treatment fails.
} 


\section{Some Results}

This section will present some results.

\section{1. "Bang-bang" solutions}

First, the existence of "bang-bang" solutions is examined. Assume the following:

$$
p_{t}=p, q_{t}=q, c_{t}=c, k_{t}=k \forall t \in\{1, \ldots, T\} .
$$

Given this assumption, the decision problem may be depicted by the decision tree in Figure 3.

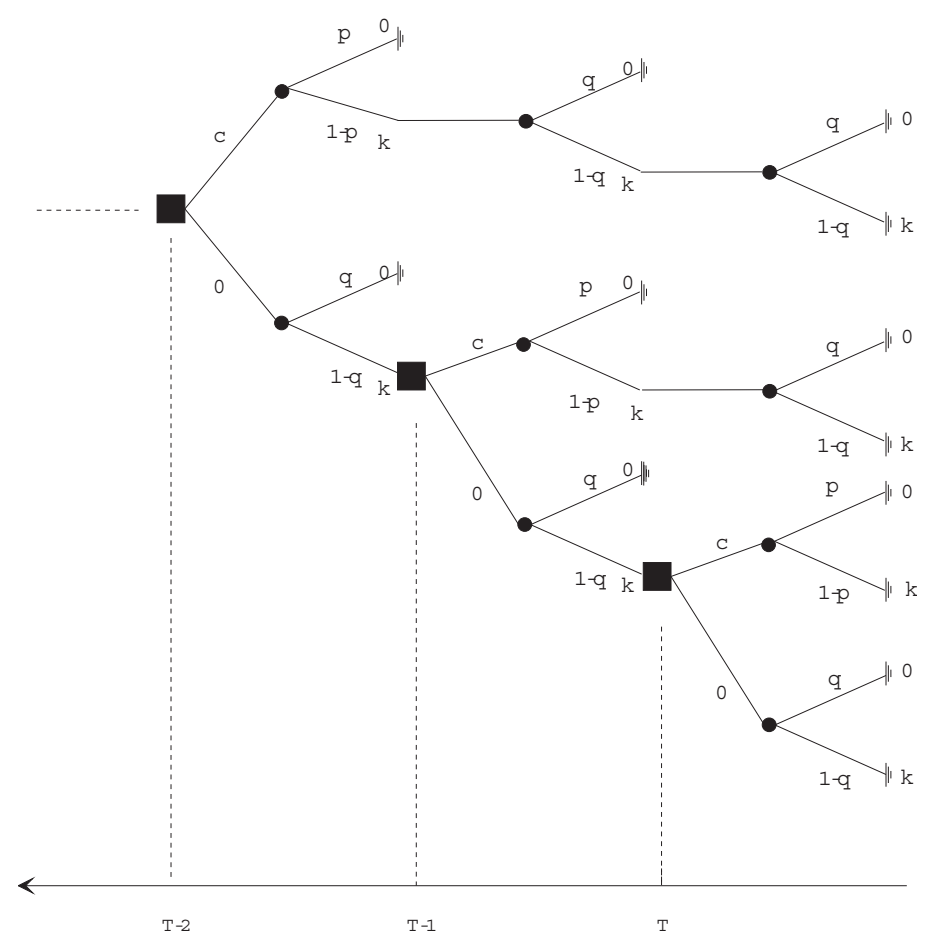

Figure 3. Decision tree with no time dependence in parameters.

Doing a backward recursion on this tree, it is easy to realize that period $T$ involves two possibilities. Either

$$
c+(1-p) k \leq(1-q) k
$$

or

$$
c+(1-p) k>(1-q) k .
$$

Let us assume that treatment is optimal in period $T$. That is

$$
\min \{c+(1-p) k,(1-q) k\}=c+(1-p) k .
$$

In period $T-1$, we obtain the following optimization problem:

$$
\min \{c+(1-p)[k+(1-q) k],(1-q)[k+c+(1-p) k]\}
$$




$$
=(1-p)(1-q) k+\min \{c+(1-p) k,(1-q) k+(1-q) c\} .
$$

Comparing Equations (3.1) and (3.3) it is straightforward to realize that treatment is optimal in period $T-1$ as well. Continuing this type of argument leads to the following decision problem in period $j$ (counted backwards)

$$
\min \left\{c+(1-p) k\left(\sum_{i=0}^{j}(1-q)^{i}\right),(1-q)\left[k+c+(1-p) k\left(\sum_{i=0}^{j-1}(1-q)^{i}\right)\right] \cdot\right\}
$$

Equation (3.4) can be written as

$$
(1-p) k\left(\sum_{i=1}^{j}(1-q)^{i}\right)+\min \{c+(1-p) k,(1-q) k+(1-q) c\} .
$$

If Equations (3.5) and (3.3) are compared, it is easy to conclude the following. Given that treatment is optimal in period $T$, it is also optimal in any earlier period $j$ including period 1 . Hence, this situation implies immediate treatment as the optimal strategy. The other possible alternative in period $T$, Equation (3.2) may either be expanded similarly or the following argument may be applied.

The optimal action in period $T$ is now not to treat. In period $T-1$, two possibilities exist; either treatment is conducted or not. If treatment is the optimal decision, this will (by the reasoning above) again be the optimal decision in period $T-2$ and so on and the result from Equation (3.5) can again be applied yielding that immediate treatment is the optimal action. Alternatively, if treatment is not the optimal action in period $T-1$, we can repeat the same argument again in period $T-2$ etc. If we end up (in period 1) with no treatment as the optimal action, the optimal treatment time is never.

The conclusion is: If $p, q, c, k$ are assumed constant, the basic result of Gafni and Mehrez - a "bang-bang" solution structure holds in this model as well.

\subsection{Existence of an interior optimum}

Following Gafni and Mehrez, it is now a straightforward matter to identify further (and more general situations) for "bang-bang" solutions. This does, however, not give much of an insight into the problem. Hence, the last part of the paper is devoted to the more interesting case with existence of interior optima.

Assume that the treatment probabilities are increasing over time ${ }^{5}$. That is

$$
p_{1}<p_{2}<\ldots<p_{T} .
$$

Given this assumption, the decision problem in period $T$ (given that treatment is optimal) may be written:

$$
\min \left\{c+\left(1-p_{T}\right) k,(1-q) k\right\}=c+\left(1-p_{T}\right) k .
$$

In period $T-1$ the decision problem becomes:

$$
\min \left\{c+\left(1-p_{T-1}\right)[k+(1-q) k],(1-q)\left[k+c+\left(1-p_{T}\right) k\right]\right\} .
$$

\footnotetext{
${ }^{5}$ Such a situation may indicate developing medical technology and methods, and seems like a very reasonable assumption.
} 
Equation (3.8) is now expanded in more detail. First, the left part within the minimization:

$$
c+\left(1-p_{T-1}\right) k+\left(1-p_{T-1}\right)(1-q) k
$$

and then the right part:

$$
(1-q) k+(1-q) c+\left(1-p_{T}\right)(1-q) k .
$$

It is readily observed that the expression $c+\left(1-p_{T-1}\right) k$ in $(3.9)$ is increasing compared to the expression $c+\left(1-p_{T}\right) k$ in (3.7). At the same time (using (3.6) again)

$$
\left(1-p_{T-1}\right)(1-q) k>\left(1-p_{T}\right)(1-q) k
$$

while the last part of $(3.10)((1-q) k+(1-q) c)$ is unchanged. As a consequence, the left part of the minimization is increasing more than the right part within the minimization - the treatment strategy is gradually becoming more and more costly compared to the not treatment strategy ${ }^{6}$. Sooner or later, provided the time horizon is long enough, the no treatment strategy will become less expensive and the interior optimal solution will occur at this point in time. Using the reasoning behind Equations (3.4) and (3.5), the optimal time of treatment $t^{*}$ may be found by solving the following equation:

$c+\left(1-p_{T-t^{*}}\right) k\left\{\sum_{i=1}^{t^{*}}(1-q)^{i}\right\}=(1-q)\left(k+c+\left(1-p_{T-t^{*}+1}\right) k\left\{\sum_{i=1}^{t^{*}-1}(1-q)^{i}\right\}\right)$.

This Equation (3.12) can easily be solved numerically. Alternatively, if $p_{t}$ can be expressed with a direct functional representation, we may find a direct analytic solution to the problem by applying the formula for the sum of a finite geometric series.

\subsection{An approximate solution}

None of the above mentioned strategies give a direct insight into how the solution behaves. As a consequence, an approximation will be presented which is intuitive and simple to calculate.

Assume that $p_{t}$ does not change much over time. That is

$$
p_{i} \approx p_{i+1} \forall i \in\{1, \ldots, T\} .
$$

Returning to Equation (3.11) and utilizing Assumption (3.13), it is easy to see that the left and right side of (3.11) will become reasonably equal. As a consequence, the minimization (3.8) may be approximated by:

$$
\min \left\{c+\left(1-p_{T-1}\right) k,(1-q) k+(1-q) c\right\} .
$$

A straightforward inductive argument then leads to the following approximate equation to determine the optimal treatment time $t^{*}$.

$$
c+\left(1-p_{T-t^{*}}\right) k \approx(1-q) k+(1-q) c .
$$

Solving (3.14) yields:

$$
p_{T-t^{*}}=q\left(1+\frac{c}{k}\right) .
$$

\footnotetext{
${ }^{6}$ Note that this arguing is done backwards.
} 
It is straightforward to justify the intuition in Equation (3.15). If the probability of being cured without treatment $q$ increases, $p_{T-t^{*}}$ increases and the optimal treatment time is postponed. Similarly, if the treatment cost $c$ increases, the same effect occurs. However, if the cost of staying ill $k$ increases, $p_{T-t^{*}}$ decreases and the optimal treatment time decreases.

\subsection{An example}

A simple example is formulated in order to show how Equation (3.15) may be used. Data for the example is given in Table 1.

Table 1. An example of applying the approximate solution.

\begin{tabular}{|c|c|c|c|c|c|c|c|c|c|c|c|c|c|c|c|}
\hline \hline Period & 1 & 2 & 3 & 4 & 5 & 6 & 7 & 8 & 9 & 10 & 11 & 12 & 13 & 14 & 15 \\
\hline$p_{t}$ & .25 & .3 & .35 & .4 & .45 & .5 & .55 & .6 & .65 & .7 & .75 & .8 & .85 & .9 & .95 \\
$q$ & .8 & .8 & .8 & .8 & .8 & .8 & .8 & .8 & .8 & .8 & .8 & .8 & .8 & .8 & .8 \\
$c$ & 0 & 0 & 0 & 0 & 0 & 0 & 0 & 0 & 0 & 0 & 0 & 0 & 0 & 0 & 0 \\
$k$ & 1 & 1 & 1 & 1 & 1 & 1 & 1 & 1 & 1 & 1 & 1 & 1 & 1 & 1 & 1 \\
\hline \hline
\end{tabular}

Equation (3.15) gives:

$$
p_{T-t^{*}}=0.8\left(1+\frac{0}{1}\right)=0.8
$$

Hence, the approximate optimal treatment time for this example is period 3 as $p_{12}=0.8$ and $15-t^{*}=12 \Rightarrow t^{*}=3$ The approximation may be controlled as $p_{t}$ may be written (see Table 1 ):

$$
p_{t}=0.20+0.05 t
$$

That is, a functional representation for $p_{t}$ is available and Equation (3.12) may be applied directly. Inserting $\sum_{i=1}^{t^{*}}(1-q)^{i}=\frac{1-q}{q}\left[1-(1-q)^{t^{*}}\right]$, Equation (3.16) and the data from Table 1 into Equation (3.12) we obtain

$$
\frac{1}{4}\left(0.05+0.05 t^{*}\right)\left[1-0.2^{t^{*}}\right]=0.2\left(1+\left[\frac{1}{4} 0.05 t^{*}\left(1-0.2^{t^{*}-1}\right)\right]\right) .
$$

The left hand minus the right hand side of (3.17) is plotted in the same diagram in Figure 4.

As Figure 4 shows, Equation (3.17) has a solution slightly above 12. As Equation (3.17) is a continuous representation of a discrete problem, this value must be rounded down yielding 12 as the correct optimal treatment time ${ }^{7}$. Surely, the difference from the approximate $t^{*}$ of 3 is striking. However, this result is not very surprising, as our underlying assumption in Equation (3.13) shows limited correspondence with the example data from Table 1 . The $p_{t}$ 's there do indeed change

\footnotetext{
${ }^{7}$ This value may of course be controlled by actually doing direct calculations on the decision tree.
} 


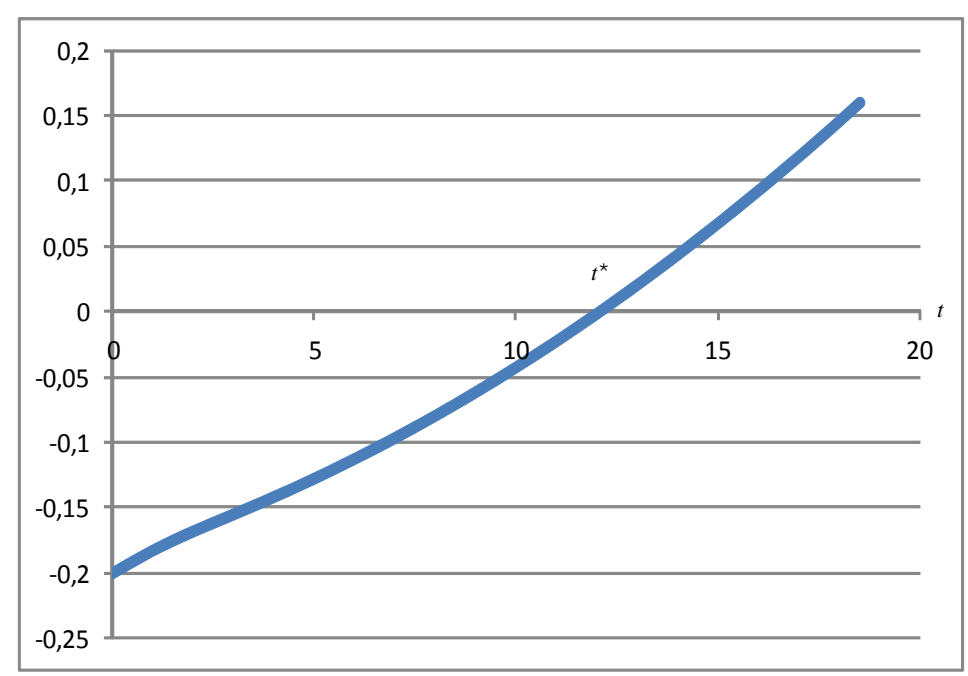

Figure 4. Optimal treatment time in the example.

a great deal - in fact a $20 \%$ increase between all periods. Hence, our example is not meant to reflect reality ${ }^{8}$, only to show how the two methods work.

\section{Conclusions}

This paper has demonstrated the existence of both "bang-bang" solutions and interior optima in a model with the aim of obtaining an optimal time of treatment for patients with musculoskeletal diseases. The modelling framework differs somewhat from similar work in the literature, but similarities exist.

The basic findings in this work are the actual conditions for the existence of an optimal treatment time as well as a simple and intuitive approximate solution.

The possibility of applying such a model in practice has not been judged, but, as SHD has granted money for the task, one would at least assume that the problem is interesting. Implementing a patient treatment strategy different from todays' is of course difficult. It can, for instance, be assumed that few doctors would welcome a model telling them which patients to treat and when. Additionally, if such a model is to be applied in a socio-economic framework, the problem of estimating the costs involved with an individual staying ill within a period may be difficult. Some relatively recent research supporting such an estimation process may, however, be helpful $[11,12]$.

\footnotetext{
${ }^{8}$ Note also the $q$-value in Table 1 which indicates that getting rid of the disease without treatment is much more probable than through treatment - hardly a very realistic assumption.
} 


\section{REFERENCES}

[1] R. J. Barro and J. W. Friedman, On uncertain lifetimes, J. Polit. Econ. 85 (1977), 843-849. DOI: $10.1086 / 260603$

[2] A. Gafni and D. Peled, The effect of labelling in illness related absenteeism, J. Helath Econ. 3 (1984), 1973-1978. DOI: 10.1016/0167-6296(84)90005-5

[3] M. W. Lund, K. Rognstad, T. Rundmo and P. Ø Saksvik, Costs Related to Industrial Accidents and Work Related Health Failure, SINTEF-report STF83 A92017, 1992.

[4] A. Mehrez and A. Gafni, The Optimal Treatment Strategy - a Patient's Perspective Address, Working Paper No. 87485, The Israel Institute of Business Research, Faculty of Management, Tel Aviv, Israel, 1985.

[5] A. Mehrez and A. Gafni, The optimal treatment strategy - a patient's perspective, Manage. Sci. 33 (1987), 1602-1612. DOI: 10.1287/mnsc.33.12.1602

[6] Challenges in health promotional and preventive work, Norwegian Parliament White Paper No. 37 (in Norwegian), 1992-1993.

[7] Note from the Coordinating Group in SHD Working with Musculoskeleteal Diseases and Rehabilitation, a Judgement of Experience and Suggestions for Further Work, Internal Report in The Ministry of Health and Social Affairs, February 1995.

[8] em The welfare message, SHD White Paper No. 35 (in Norwegian), 1994-1995.

[9] J. E. Stiglitz, Economics of the Public Sector, 2nd ed., W. W. Norton \& Company Inc., New York, 1988.

[10] The states Revenues and Expenditures, The Norwegian Ministry of Finance (in Norwegian), http: //www.regjeringen.no, July 2013.

[11] G. W. Torrance, D. L. Sackett and W. H. Thomas, A utility model for evaluation of health care programs, Health Serv. Res. 7 (1972), 118-133.

[12] G. W. Torrance, M. H. Boyle and S. P. Horwood, Application of multi-attribute utility theory to measure social preferences for health states, Oper. Res. 30 (1982), 1043-1069.

[13] M. E. Yaari, Uncertain lifetime, life insurance and the theory of consumer, Rev. Econ. Stud. 33 (1965), 137-150. DOI: 10.2307/2296058

Kjetil K. Haugen, Faculty of Economics, Informatics and Social Science, Molde University College, Box 2110, 6402 Molde, Norway

e-mail: kjetil.haugen@himolde.no 